\title{
Activation by interferon- $\gamma$ of expression of ICAM-1 and MHC class II antigens in tumour cells from colorectal carcinomas
}

\author{
I Donnellan, J Cantrill, I Fraser, A Morris
}

\begin{abstract}
Aims-To determine whether lack of MHC class II antigen and intercellular adhesion molecule-1 (ICAM-1) expression in some tumours is due to the inability of the tumour cells to respond to the cytokine interferon- $\gamma$ (IFN- $\gamma$ ), an important activator of these surface molecules.

Methods-Cells from 40 colorectal tumours which did not constitutively express class II MHC antigens or ICAM-1 were kept in short term culture after disaggregation for a few days to two weeks without significant loss of viability. These were treated with IFN- $\gamma$. Expression of class II MHC antigens and ICAM-1 was determined using immunohistological techniques.
\end{abstract}

Results-There was clear induction in vitro of both MHC class II antigens and ICAM-1 in cells from eight of the tumours, with between 50 and $80 \%$ of the tumour cells in the cultures staining positively. The staining was apparent within 24 hours, appeared maximal at about three days, and declined thereafter. There were no obvious differences in cell morphology or viability between the cultures which were inducible and those which were not, nor were there obvious differences between the tumours from which they were derived. Conclusions-Expression of MHC class II antigens and ICAM-1 may be induced by IFN- $\gamma$ in a small proportion of colorectal tumours which do not constitutively express these antigens, showing that only a minority of tumours are capable of responding to this cytokine.

(f Clin Pathol: Mol Pathol 1995;48:M40-M45)

Keywords: MHC class II antigens, ICAM- 1 , interferon- $\gamma$, colorectal tumours.

Walsgrave Hospital NHS Trust, Clifford Bridge Road, Coventry CV2 2DX

I Donnellan

J Cantrill

I Fraser

Department of Biological Sciences, University of Warwick, Coventry CV4 7AL, A Morris

Correspondence to: Miss I Donnellan, Warwick Hospital, Lakin Road, Warwick, CV34 5BW.

Accepted for publication 21 November 1994 \begin{abstract}
cinomas, ${ }^{3}$ lung carcinomas ${ }^{4}$ and colorectal carcinomas, ${ }^{56}$ amongst others, express tumour antigens which are recognised specifically by $T$ lymphocytes.

is accumulating evidence that cifically in the context of major histolympatibility complex (MHC) antigen from the study of melanoma, where target antigens and $\mathrm{MHC}$ restriction elements have been identified. ${ }^{12}$ Evidence for other tumours is less
\end{abstract}

T lymphocyte recognition of tumour neo-antigens requires that these be complexed to $\mathrm{MHC}$ antigens in so-called MHC restricted recognition. ${ }^{7}$ An alternative non-MHC restricted pathway exists which relies on cell adhesion molecules such as intercellular adhesion molecule-1 (ICAM-1), a ligand for lymphocyte function associated antigen-1 (LFA-1) ${ }^{8-10}$ ICAM-1 is also necessary for efficient binding of $\mathrm{T}$ cells to targets of MHC restricted effector cells. ${ }^{7112}$ Tumour cells lacking these molecules at their surfaces will not be recognised by T lymphocytes and so a potentially immunogenic tumour could avoid surveillance. Direct experimental evidence for this has been obtained from a number of mouse models. ${ }^{1314}$

Loss of class I MHC antigens (HLA A, B, C), normally expressed by bowel epithelium, is common in colorectal carcinoma, with either single alleles or occasionally all alleles missing. ${ }^{15-17}$ This loss of class I MHC antigen expression by human tumours has been taken as indirect evidence for antitumour mechanisms mediated by specific $T$ lymphocytes as the loss implies immune selection of class I negative cells. However, class II antigens (HLA DR, DP, DQ), not normally expressed by epithelium are expressed by malignant colonic epithelium with about one third of tumours. ${ }^{15161819}$ As with class II antigens, ICAM-1 is not expressed on normal bowel epithelium but is reported to be variably expressed by malignant colonic epithelium..$^{20}$

Both MHC class II antigens and ICAM-1 are overexpressed in areas of inflammation, in autoimmune disease and allograft rejection. In rejecting kidneys, for example, there is increased expression of both MHC class II antigens and ICAM-1 by tubular cells as well as endothelial cells, accompanied by heavy infiltration of activated leucocytes. ${ }^{21}$ Cytokines, especially interferon- $\gamma$ (IFN- $\gamma$ ), which are the products of inflammatory cells, induce or augment the expression of both MHC class II antigens and ICAM-1. ${ }^{2223}$ The likely mechanism for activation of MHC class II antigens and ICAM-1 in inflammatory conditions is local production of cytokines and this is assumed to be the case for tumours also. However, many colorectal carcinomas fail to express MHC class II antigens and ICAM-1, despite the presence of inflammatory cells. There are two possible explanations for this: either there is insufficient local production of cytokines or the malignant cells are incapable of responding to cytokines. We undertook this study to address the latter possibility. 
At least some colorectal tumour cell lines are able to express both MHC class II antigens and ICAM-1, but results with long term lines are suspect because such lines are selected for in vitro growth and so may not be representative of the original tumour. Also, they are released from the influence of stromal factors, such as inhibitory cytokines, which might prevent expression in vivo. We have therefore treated tumour cells, derived from human colorectal tumours negative for expression MHC class II antigens and ICAM-1, in short term culture with IFN- $\gamma$ to determine whether they can be induced to express MHC class II antigens and ICAM-1.

\section{Methods}

TUMOUR SPECIMENS AND CELL CULTURE

Fresh samples of tumour tissue, macroscopically normal colon and samples of colon affected by ulcerative colitis, were obtained from patients undergoing surgical resection. In the patients with cancer the "macroscopically normal" colon was obtained from a site at least $10 \mathrm{~cm}$ from the tumour. Six samples were obtained during colectomy for severe ulcerative colitis. All the specimens were washed individually in sterile RPMI 1640 medium (Gibco BRL Life Technologies, Paisley, UK) before being placed on ice in complete medium (RPMI 1640 supplemented with $2.0 \mathrm{mM} \mathrm{L-}$ glutamine, $100 \mathrm{U} / \mathrm{ml}$ penicillin/streptomycin, $2.5 \mathrm{mg} / \mathrm{ml}$ gentamicin, and $10 \%$ fetal calf serum. A portion of each specimen was snapfrozen in liquid nitrogen and stored at $-70^{\circ} \mathrm{C}$ until immediately prior to sectioning. Sequential $6 \mu \mathrm{m}$ thick sections of normal and diseased tissue were cut and placed on the same slide, fixed in cold acetone for 10 minutes, air-dried and stored at $-70^{\circ} \mathrm{C}$ until staining. The remaining tumour was washed several times in RPMI 1640 and any debris carefully removed. It was minced into $1 \mathrm{~mm}^{3}$ pieces and enzymatically disaggregated in a solution of complete medium supplemented with $0.01 \mathrm{mg} /$ $\mathrm{ml}$ hyaluronidase type $\mathrm{V}, 0.02 \mathrm{mg} / \mathrm{ml}$ DNAse type I, and $1 \mathrm{mg} / \mathrm{ml}$ collagenase type IV (Boeringer-Mannheim, Lewes, UK) for 12 to 15 hours at room temperature. The cells were washed three times by centrifugation in complete medium and then cultured in complete medium at $37^{\circ} \mathrm{C}$ in $5 \% \mathrm{CO}_{2}$ enriched air. Cultures were supplemented with $100 \mathrm{U} / \mathrm{ml}$ recombinant human IFN- $\gamma,{ }^{24} 10 \mu \mathrm{M} / \mathrm{ml}$ retinoic acid (Sigma Chemical Co., Poole, UK), or $10 \mu \mathrm{l} / \mathrm{ml}$ recombinant human tumour necrosis factor- $\alpha$ (TNF- $\alpha$ ) (R\&D Systems Europe, Abingdon, UK): control cultures were not supplemented. Cell viability was assessed daily using the trypan blue exclusion test. Only cultures with $>65 \%$ viability and no microbial contamination were studied. The medium in the tissue cultures was changed twice weekly. Cytospin slide preparations (Shandon Corporation, Runcorn, UK) of the tumour cell preparations were made after 24,48 and 72 hours of incubation. Care was taken to ensure that only cells in suspension were taken in order to reduce the likelihood of harvesting fibroblasts which could not be totally eliminated from such short term culture preparations. The slides were air-dried, fixed in cold acetone and stored as described previously.

\section{IMMUNOHISTOCHEMICAL STAINING}

The sections and cytospin preparations of cultured cells were stained using an avidin-biotin technique. The slides were permitted to warm to room temperature. Endogenous peroxide activity was quenched by immersion in 3\% hydrogen peroxide in methanol solution for five minutes. The slides were rinsed in phosphate buffered saline (PBS) $(\mathrm{pH} \mathrm{7.4)}$ before incubation with the pimary antibody (all murine monoclonal antibodies).

These were antibody to ICAM-1 (CD25) clone $84 \mathrm{H} 10$ (Serotec MCA532, Oxford, UK); three antibodies recognising human class II framework determinants, clone WR18 recognising determinants common to HLA DR, DP, and DQ (Serotec MCA532), clone CR3/43 recognising a DR specific determinant (Dako, High Wycombe, UK, M775) and L243 also recognising a DR specific determinant (Becton Dickinson, Oxford, UK, 347360); antibody recognising a framework determinant of human HLA A, B, C clone W6/32 (Dako M736); anticarcinoembryonic antigen (CEA) clone 11-7 (Dako M70702); anticytokeratin clone MNF116 (Dako M821); anti-CD3 clone T34B5 (Dako M716); and anti-interleukin-2 receptor (CD25) clone ACT-1 (Dako M731).

The sections were rinsed and the secondary biotinylated rabbit antimouse antibody (Dako) added. Incubation with the ABC solution (Dako) followed, and the final chromagen was $\mathrm{DAB}\left(3,3^{\prime}\right.$ diaminobenzidine tetrachloride; $\mathrm{BDH}$, Lutterworth, UK). The slides were counter-stained with Meyer's haematoxylin $(\mathrm{BDH})$, before mounting in DPX $(\mathrm{BDH})$. Frozen sections of human tonsil were used as positive controls throughout the staining procedures: for negative controls, the primary antibody was omitted. Histological confirmation and staging of tumours were carried out on paraffin wax sections stained with haematoxylin and eosin. All slides were assessed by the investigators and reported by experienced histopathologists (Dr J Crocker, Heartlands NHS Trust Hospital and Drs T Ashworth and K Chen, Walsgrave NHS Trust Hospital).

\section{Results}

\section{TISSUE SAMPLES}

Carcinoma was histologically confirmed in all 50 cases. Of these, six were well differentiated, 38 moderately differentiated, six poorly differentiated, and two of a mucinous type. In six cases the tumour was known to have metastasised to the liver at the time of surgery, in three the tumour was confined to the mucosa (Dukes' stage A), in 20 it had extended through the bowel wall (Dukes' stage B), and in 21 adjacent lymph nodes contained tumour deposits (Dukes' stage $C$ ). In six of the 50 cases there was evidence of a pronounced lympho- 

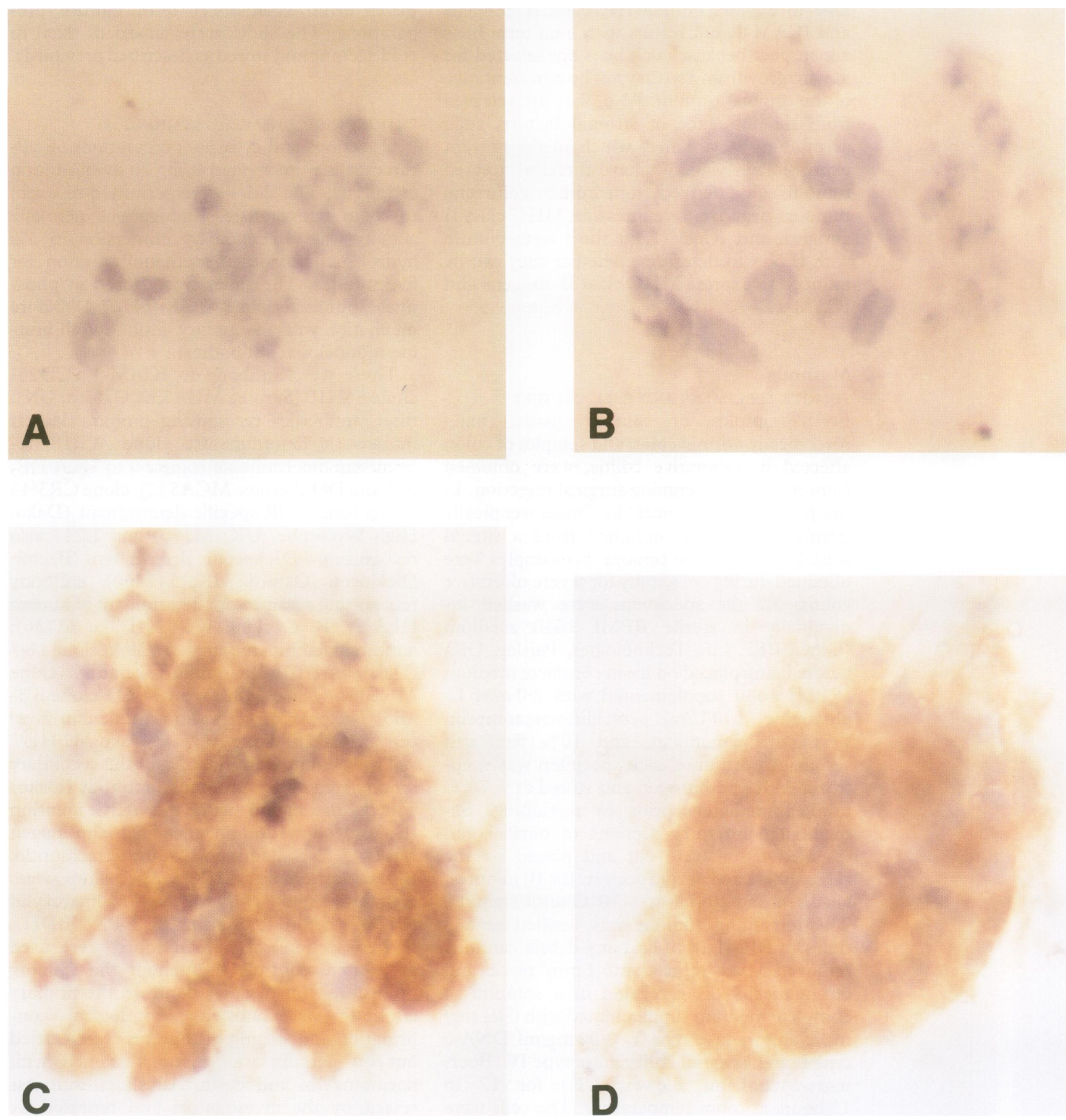

Figure 1 Tumour cells were treated and stained after two days of culture.

A: Control cells (untreated) stained for class II antigen; B: control cells (untreated) stained for ICAM-1;C: IFN- $\gamma$ treated cells stained for class II antigen; $D:$ IFN- $\gamma$ treated cells stained for ICAM-1 (magnification $\times 400)$.

cytic infiltration, but lymphocytes were seen in sections from all of the tumours. The "normal" tissue taken from these patients showed no evidence of tumour cell infiltration. $\mathrm{Mi}$ croscopic examination of the samples of tissue obtained from six patients with ulcerative colitis showed the typical features of ulcerative colitis with severe inflammation, associated with a heavy inflammatory cell infiltrate, crypt abscess formation and some epithelial dysplasia. None of these specimens showed any evidence of malignancy.

EXPRESSION OF MHC ANTIGENS AND ICAM-1 BY TUMOURS AND OTHER TISSUES

The constitutive MHC and ICAM-1 ex- pression by the original tumours was investigated in tissue sections. Staining of tumour sections for MHC class I antigens showed these to be present in all three cases in which staining was absent either completely or in parts of the tumour. However, staining with the anti-class II antibodies showed that in no case was there general or even extensive expression by the malignant glandular epithelium cells, although vascular endothelium, infiltrating inflammatory cells and Peyer's patches all stained strongly within the same section. In occasional tumours a few of the malignant cells stained weakly. This was a surprising result: there have been several previous reports that colorectal tumours express MHC class II antigens and in a series 
of 50 we expected several positive results. On the other hand, in four samples the "normal" colonic mucosa derived from regions of the colon close to the tumour exhibited class II staining of the epithelial cells. Also, all of the tissue samples from all six cases of ulcerative colitis expressed class II antigens. In all of these cases a leucocytic infiltrate was present.

Similarly, malignant mucosal cells did not stain for ICAM-1 in any of the sections although again vascular endothelium, infiltrating inflammatory cells and Peyer's patches all stained clearly. The samples of "normal" bowel positive for class II antigens, were also ICAM1 positive, as were the samples from patients with ulcerative colitis.

\section{SHORT TERM CULTURES OF TUMOUR CELLS}

Cells in suspension cultures derived from 40 of the 50 tumours remained more than $65 \%$ viable for a minimum of 72 hours as assessed by trypan blue exclusion. The remaining cultures were lost either to microbial contamination or rapid decline in cell viability. Stained cytospin preparations of the cultures demonstrated the presence of viable tumour cells, which could be confidently distinguished from leucocytes or fibroblasts as judged on morphological criteria (fig 1). Staining with antibodies to CEA or cytokeratin was also used to confirm the presence of carcinoma cells in the culture.

With most of the cultures, significant numbers of viable tumour cells persisted for many days and in some cases tumour spheroids formed. However, no attempt was made to establish growing cultures or indeed to maintain the cultures beyond the experimental period.

\section{INDUCTION OF MHC ANTIGENS AND ICAM-1 IN CULTURED TUMOUR CELLS}

There was no change in expression of class II antigens (fig 1A) or ICAM-1 (fig 1B) by tumour cells in the control cultures untreated with IFN- $\gamma$; in all cases tumour cells were negative for both class II antigens (staining with both Serotec and Dako antibodies) and ICAM-1 and positive for MHC class I antigens, confirming the results obtained with the tumour sections. On the other hand, in eight of the IFN- $\gamma$ treated cultures $(20 \%)$ there was clear staining for both class II antigens (fig 1C) and ICAM-1 (fig 1D) in cells which were identifiable as tumour cells on morphological grounds, indicating that these molecules were induced in the tumour cells. The proportion of cells expressing these antigens varied between 50 and $90 \%$ in cultures from different tumours. Expression was apparent by 24 hours and persisted for at least 72 hours: however, by one week all tumour cells in the culture were negative. In some cases (five of eight) it was possible to re-treat inducible cultures and both MHC class II antigen and ICAM-1 expression could be induced for a second time. Cells from seven adenomas were similarly cultured and treated: no induction of class II antigens or ICAM-1 was detected.

Inducible cultures were derived pre- dominantly (six of eight) from moderately differentiated Dukes' stage B carcinomas; the others were a well differentiated Dukes' stage A and a moderatively differentiated Dukes' stage C tumour. The degree of inflammatory infiltrate as seen by conventional haematoxylin and eosin staining and confirmed by CD3 and interleukin-2 receptor staining on the tissue sections did not appear to be related to the inducibility of MHC class II antigen and ICAM-1 expression. In cases where the "normal" mucosa expressed MHC class II antigens and ICAM-1 none of the tumour cells could be induced to express either molecule. Although the follow up time was short (12-24 months) five patients have died of their disease, but these patients did not have tumour cells capable of expressing MHC class II antigens or ICAM-1.

In some cases attempts were made to induce MHC class II antigens and ICAM-1 with retinoic acid on TNF- $\alpha$, but these were uniformly unsuccessful.

\section{Discussion}

Previous studies have reported that MHC class II antigens are expressed by the colonic mucosa in colorectal tumours ${ }^{16-19}$ and in some cases have been associated with the presence of infiltrating mononuclear leucocytes ${ }^{25}$. Whether or not the MHC class II or ICAM-1 phenotype influences prognosis is the subject of continued debate and there are conflicting series of follow up data. A series reported by Moller et $a l^{16}$ in 1991 did not find any supportive evidence for MHC class II antigen expression influencing the outcome of the disease, but a more recent publication by Norheim-Andersen et $a l^{19}$ appeared to suggest that the presence of MHC class II antigen expression may be correlated with a more favourable prognosis as seen in gastric cancer, ${ }^{26}$ squamous carcinoma of the laryn $x^{27}$ and breast cancer. ${ }^{28}$

In the 50 carcinomas studied here very limited constitutive MHC class II antigen expression was found, despite the presence of activated inflammatory cells in all the tumours, and heavy infiltration in six. The result was confirmed by restaining the whole series with a second and a third antibody to MHC class II antigens, one of which had been used in a previous series ${ }^{19}$ to stain MHC class II antigens in colorectal tumours. In all cases it was clear that inflammatory and other non-malignant cells within the tumour stained, thus acting an internal controls. We observed both MHC class II antigen and ICAM-1 expression in areas of "normal" mucosa adjacent to tumours and in samples of ulcerative colitis. The ulcerative colitis samples were used as a positive control, an inflammatory condition without malignancy. Also, in a series of oesophageal carcinomas stained in parallel, some six of 37 tumours clearly stained strongly with the Dako CR3/43 antibody. ${ }^{29}$ We are therefore confident that in the tumours studied here there was little or no expression of MHC class II antigens by the malignant cells.

Similarly, we found little or no ICAM-1 
expression. There is little documented with regard to the expression of ICAM-1 in colorectal cancer. In a study by Kelly et $a l^{20}$ constitutive expression was described in five of 10 cancers. However, positively staining cells were infrequent, and amounted to less than $5 \%$ of the epithelial cells in any given section. The authors considered this expression to be more prevalent in areas of lymphocytic infiltration.

The main purpose of this study was to determine whether these antigens were inducible in ex vivo tumour cells and so non-expressing tumours were the essential starting material. We found that in cultures from at least some of the tumours (20\%), both MHC class II antigen and ICAM-1 expression was inducible in the malignant cells. The expression was coordinately induced in that in tumours where MHC class II antigen expression was induced, so was ICAM-1. However, no double staining was done so we do not know whether both antigens were coordinately expressed in individual cells: this is likely in those cultures where most cells were expressing these antigens. The failure of the induction of MHC class II antigens and ICAM-1 in the cultures of the remaining tumours could potentially be due to technical reasons, such as low viability, but there were no obvious differences in viability between these cultures and the responding cultures, nor where there obvious differences in the morphology of the tumour cells, frequency of other cell types present, etc. The ability of the tumour cells to respond in vitro to IFN- $\gamma$ treatment was independent of the degree of differentiation, stage of the cancer, or the degree of inflammatory infiltration of the original tumour. We feel it is a reasonable conclusion that in some colorectal carcinomas which do not express MHC class II antigens or ICAM-1 in vivo these antigens can be induced at least by IFN- $\gamma$ but that the majority are not inducible.

In the inducible tumours the lack of in vivo expression was presumably due to the lack of sufficient local cytokine production, or production of inhibitory factors. In these tumours activated $T$ cells (positive for CD25, the interleukin-2 receptor) and macrophages were seen in all the sections examined but it cannot be assumed that they are capable of producing physiological amounts of pro-inflammatory cytokines to augment ICAM- 1 and MHC class II antigen expression, although we have found that tumour infiltrating lymphocytes are capable of producing similar amounts of IFN- $\gamma$ to peripheral blood cells from the same patient.

Failure of tumour cells to respond to IFN- $\gamma$ could be because of-for example, lack of appropriate receptors, or the presence of inhibitory factors such as TGF- $\beta$ produced by cancer cells and dampening any response. Many established colon cancer lines treated with cytokines retain the ability to respond to stimulation with the upregulation of ICAM-1 expression to a greater or lesser degree ${ }^{30}$ (and our own unpublished observations which indicate that seven of nine lines respond to IFN- $\gamma$ by expressing MHC class II antigens and ICAM-1). We found that two primary colo- rectal tumour lines, after being maintained in vitro for a few weeks, expressed HLA-DR in response to IFN- $\gamma$ to a greater degree. These observations would tend to support the hypothesis that local factors within the tumour environment limit the responsiveness of the tumour cells to cytokines, and this hyporesponsiveness may permit the tumour cells to escape both non-MHC restricted and MHC restricted lymphocyte killing, and in doing so confer a growth and metastatic advantage. This may have implications when deciding whether adjuvent therapy, possibly with cytokines, should be offered to these patients.

We are grateful to the Cancer Research Campaign for financial support

1 Wolfel T, Hauer M, Klehmann F, Brichard V, Ackermann $\mathrm{B}$, Knuth A, et al. Analysis of antigens recognised on human melanoma cells by A2 restricted cytolytic T-lymphocytes (CTL). Int f Cancer 1993;55:237-44.

2 Slingluff $C$, Cox A, Henderson R, Hunt D, Engelhard $V$. Recognition of human melanoma cells by HLA-A2.1 restricted T-lymphocytes is mediated by at least six shared restricted T-lymphocytes is mediated by at least side epitopes. F Immunol 1993;150:2955-63.

3 Schendel D, Gansbacher B, Oberneder R, Kriegmair M Hofstetter A, Riethmuller G, et al. Tumor-specific lysis of human rental-cell carcinomas by tumor-infiltrating lymphocytes. 1. HLA-A2 restricted recognition of autologous and allogeneic-tumor lines. F Immunol 1993;151:4209-20.

4 Slingluff C, Cox A, Stover J, Moore M, Hunt D, Engelhard V. Cytotoxic T-lymphocyte response to autologous human squamous cell cancer of the lung: Epitope reconstruction with peptides extracted from LHA-AW68. Cancer Res with peptides extrac

5 Ransom J, Pelle B, Hubers H, Keynton L, Hanna M, Pomato $\mathrm{N}$. Identification of colon-tumor-assocated antigens by T-cell lines derived from tumor-infiltrating lymphocytes and peripheral-blood lymphocytes from patients immunized with an autologous tumour-cell/bacillus Calmette-Guerin vaccine. Int $f$ Cancer 1993;54:734-40.

6 Townsend A, Ohlen C, Rogers M, Edwards J, Mukherjee $S$, Bastin J. Source of unique tumour antigens. Nature 1994;371:662.

7 Springer T. Adhesion receptors of the immune system. Nature 1990;346:425-34.

8 Marlin S, Springer T. Purified intercellular-adhesion molecule-1 (ICAM-1) is a ligand for lymphocyte functionassociated antigen-1 (LFA-1). Cell 1987;51:813-19.

9 Rothlein R, Dustin M, Martin S, Springer T. A human intercellular adhesion molecule (ICAM-1) distinct from LFA-1. F Immunol 1986;137:1270-4.

10 Dustin M, Springer T. Lymphocyte function associated antigen (LFA-1) interaction with intercellular adhesion molecule-1 (ICAM-1) is one of at least three mechanisms for lymphocyte adhesion to cultured endothelial cells. $f$ Cell Biol 1988;107:321-31.

11 Vanky F, Wang P, Patarroyo M, Klein E. Expression of the adhesion molecule ICAM-1 and major histocompatibility complex class I antigens on human tumor cells is required Cancer Immunol Immunother 1990;31:19-27.

12 Gregory C, Murray R, Edwards C, Rickinson A. Down regulation of cell adhesion molecules LFA-3 and ICAM-1 in Epstein-Barr virus positive Burkitt's lymphoma underlies tumour cell escape from virus specific T-cell surveillance. $\mathcal{F} \operatorname{Exp}$ Med 1988;167:1811-24.

13 Eisenbach L, Hollander N, Greenfeld L, Yakor H, Segal S, Feldman $M$. The differential expression of $H-2 K$ versus $\mathrm{H}-2 \mathrm{D}$ antigens distinguishing high metastatic from low metastatic clones is correlated with the immunogenic metastatic clones is correlated with the immunogenic properties

14 Hammerling G, Klar D, Pulm W, Momburg F, Moldenhaue G. The influence of major histocompatibility complex class I antigens on tumour growth and metastasis. Biochim Biophys Acta 1987;907:245-57.

15 McDougall C, Ngoi S, Goldman I, Felix J. Reduced expression of HLA class I and II antigens in colon cancer. Cancer Res 1990;50:8023-7.

16 Moller P, Momberg F, Koretz K, Moldenhauer G, Herfarth $\mathrm{C}$, Otto $\mathrm{H}$, et al. Influence of major histocompatibility complex class $I$ and II antigens on survival in colorectal cancer. Cancer Res 1991;51:729-36.

17 Kaklamanis L, Gatter K, Hill A, Mortensen N, Harris A Krausa P, et al. Loss of HLA class I alleles, heavy chains and beta-2 microglobulin in colorectal cancer. Int $\mathcal{f}$ Cancer and beta-2 microglot.

18 Garrido F, Cabrera T, Concha A, Glew S, Ruizcabello F Stern P. Natural history of HLA expression during tumour Stern P. Natural history of HLA expression during tumour development. Immunol Today 1993;14:491-9.

19 Norheim-Andersen S, Rognum T, Lund E, Meling G Hauge S. Strong HLA-DR expression in large bowel carcinomas is associated with good prognosis. $\mathrm{Br} \mathcal{F}$ Cancer 1993;68:80-5.

20 Kelly CP, O'Keane J, Orellana J, Schroy P, Yang S, Lamon $\mathrm{J}$, et al. Human colon cancer cells express ICAM-1 in vivo 
and support LFA-1 dependent lymphocyte adhesion in vitro. Am 7 Physiol 1992;263:G864-70.

21 Andersen C, Ladefoged S, Larsen S. Acute kidney graft rejection-a morphological and immunohistological study on zero-hour and follow-up biopsies with special emphasis on zero-hour and follow-up infiltrates and adhesion molecules. APMIS 1994;102:23-37.

22 Glimcher L, Kara C. Sequences and factors: a guide to MHC class II transcription. Annu Rev Immunol 1992;10 13-49.

23 Rothlein R, Czajkowski M, O’Neill M, Marlin S, Mainolf $\mathrm{E}$, Merluzzi V. Induction of ICAM-1 on primary and continuous cell lines by pro-inflammatory cytokines. $\mathcal{F}$ Immunol 1988;141:1665-9.

24 Morris A, Ward G. Production of recombinant interferons by expression in heterologous mammalian cells. In: Clemens M, Morris A, Gearing A, eds. Lymphokines and mens M, Morris A, Gearing A, eds. Lymphokines and
interferons: a practical approach. Oxford: IRL Press, 1987: interferons:

25 Allen C, Hogg N. Association of colorectal epithelium ex- pressing HLA DR with $\mathrm{CD}^{+}$T-cells and mononuclear phagocytes. Cancer Res 1987;47:2919-23.

26 Hilton $D$, West $K$. An evaluation of the prognostic significance of HLA DR expression in gastric carcinoma. nificance of HLA DR expression in gastric carcinoma.
Cancer 1990;66:1154-7.

27 Esteban F, Ruizcabello F, Concha A, Perezayala M, Sanchezrozas J, Garrido F. HLA DR expression is associated with excellent prognosis in squamous cell carcinoma of the larynx. Clin Exp Metastasis 1990;8:319-28.

28 Concha A. Tumour aggressiveness and MHA class I and II antigens in laryngeal and breast cancer. Cancer Biol 1991; 2:47-54.

29 Rockett JC, Darnton SJ, Crocker J, Matthews HR, Morris AG. Expression of HLA-ABC, HLA-DR and intercellular adhesion molecule- 1 in oesophageal carcinoma. $f$ Clin Pathol 1995; in press.

30 Kaiserlian D, Rigal D, Abello J, Revillard J. Expression, function and regulation of the human adhesion molecule-1 (ICAM-1) on human intestinal epithelial cell lines. Eur $\mathcal{f}$ Immunol 1991;21:2415-21. 